\title{
Masks Mask Communication - Communicating with Children in Health Care Settings
}

\author{
Divya Swaminathan $^{1} \cdot$ Shoba S. Meera ${ }^{1}$ \\ Received: 24 June 2020 / Accepted: 7 October 2020 / Published online: 20 October 2020 \\ (C) Dr. K C Chaudhuri Foundation 2020
}

To the Editor: Communication, the process of exchanging information, plays an important role in social interactions and helps establish and maintain most relationships including patient-clinician relationships. Communication has two components: verbal (speech sounds) and non-verbal (gestures, body language and facial expression). In view of the COVID-19 pandemic, use of face masks is recommended $[1,2]$. However, face masks hamper communication, both verbal (e.g., attenuates speech signals produced by the speaker thus degrading quality of speech) and non-verbal mode (e.g., eliminates large part of facial expressions). While some environments make for better communication even with the masks on, (e.g., when the content is known - communicating with a cashier in a grocery store), some can be harder (e.g., talking to a stranger, visiting a physician's clinic). Using face masks and communicating with children can be challenging. For communication to be effective, it must take place in a manner appropriate to one's age, understanding and communication abilities. As speech-language pathologists working with chil- dren with communication disorders, our transition to the new normal of communicating with face masks was quick and smooth. We present communication strategies (Fig. 1) we believe will be helpful for pediatric clinicians when wearing masks. While some of these strategies are similar to those, we have recommended for clinicians communicating with adults with neuro-psychiatric disorders [3], others are specifically useful when communicating with children (e.g., use of social stories). In addition to the more easily adaptable verbal strategies, we have provided non-verbal strategies that include communication using gestures, body language, pictures and written modes. We have also included tips for preparing a child for a clinic visit. Communication with children can be mediated through parents.

With the increase in active COVID-19 cases in the country, use of masks is expected to continue for the foreseeable future. Additionally, more families are bringing in children for clinical services, including non-emergency cases. Hence, equipping oneself with various communication strategies, being
Shoba S. Meera

ssmeera@nimhans.ac.in; ssmeeras@gmail.com

1 Department of Speech Pathology and Audiology, National Institute of Mental Health and Neuro-Sciences (NIMHANS),

Bangalore, India 


\section{Verbal strategies}

- Communicate effectively (e.g. ask your questions and then pause). The tone of voice must be sound friendly and reassuring. Vary in tonation to convey emotions.

- Provide time to get answers and do not overwhelm children with a lot of questions.

- Clinicians must remember to be patient and to communicate using simple sentences accompanied with gestures.

- Keep your voice even, tone gentle, and do not speak fast.

- Manage loudness - be loud, but sound reassuring.

- Clinicians must try to avoid open ended questions (e.g. ask yes/no questions)

- Repeat important words and phrases.

\section{Non-verbal strategies}

- Greet children with a gesture accompanying the word 'hello' or use a finger puppet to say hello.

- Use finger signs for encouragement (e.g. thumbs up).

- Point to things

- Use gestures to mimic what you say (e.g. gesture the action sleep when talking about sleep issues).

- Nod to acknowledge you are listening and understanding.

- Stay at the level of the child and maintain a good posture. Non-verbal cues should send message of kindness and empathy.

- Print emojis to have them handy or even use a very small dry erase pad to draw sad faces, happy faces, etc. Keep it simple!

- Use topic-based communication boards and videos to explain medication.

- Use a body chart in case the child wants to point where the pain is.

\section{Prepare the child for a clinic visit}

- Parents must be told to explain the purpose of visit and how things may be different at the doctor's clinic (e.g. use of masks, physical distancing, change in environment ). Parents can use social stories to explain these things. Social stories can be of particularly help for children with developmental disabilities (e.g. autism spectrum disorder).

- If the appointment is pre-planned, then the consultant can send a picture of themselves with and without wearing a mask/face shield prior to the appointment, making sure the child is prepared ahead of time as to who they will see.

- Parents can carry kit bag with toys, books to keep the child engaged since toys won't be available in the clinic/hospital. It should also contain food and water.

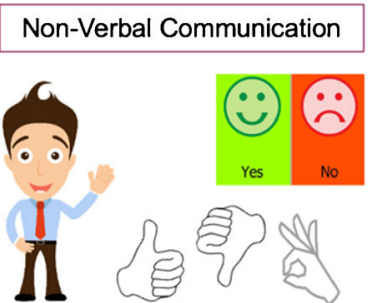

\section{Communication Board}
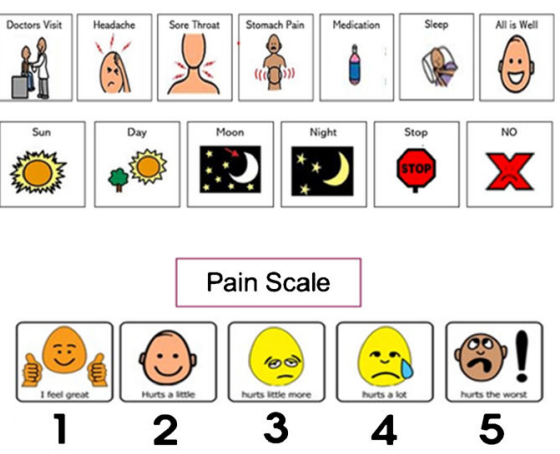

Prepare the child

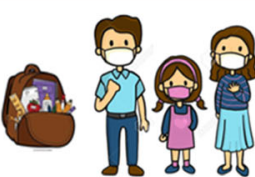

Fig. 1 Communication strategies for clinicians working with children when wearing masks

aware of the power of non-verbal communication, reminding oneself to be patient with children are crucial for patient care in these difficult times.

Acknowledgements We thank Nikita Dadlani, Prathiksha Vaidhyanathan and Dr. Prabha Chandra, Professor of Psychiatry, NIMHANS, for their valuable inputs in putting together communication strategies.

\section{Compliance with Ethical Standards}

Conflict of Interest None.

\section{References}

1. Klompas M, Morris CA, Sinclair J, Pearson M, Shenoy ES. Universal masking in hospitals in the COVID-19 era. N Engl J Med. 2020;382:e63.

2. Leung CC, Lam TH, Cheng KK. Mass masking in the COVID-19 epidemic: people need guidance. Lancet. 2020;395:945.

3. Vaidhyanathan P, Dadlani N, Meera SS, Chandra PS. Communication beyond barriers - effective communication with individuals with neuropsychiatric disorders when wearing masks. Asian J Psychiatr. 2020;54:102286.

Publisher's Note Springer Nature remains neutral with regard to jurisdictional claims in published maps and institutional affiliations. 